\title{
İki Boyutlu Koordinat Dönüşümünde En Küçük Kareler ve Toplam En Küçük Kareler Yöntemlerinin Performansı
}

\author{
Bahattin ERDOĞAN ${ }^{1}$, Utkan Mustafa DURDAĞ $\breve{~}^{2}$, Ali Hasan DOĞ $\breve{N^{3}}$, Taylan ÖCALAN ${ }^{4 *}$ \\ ${ }^{1}$ Yıldız Teknik Üniversitesi, İnşaat Fakültesi, Harita Mühendisliği Bölümü, İstanbul \\ (berdogan@yildiz.edu.tr) ORCID ID 0000 - 0002 - 8060 - 9208 \\ ${ }^{2}$ Yıldız Teknik Üniversitesi, İnşaat Fakültesi, Harita Mühendisliği Bölümü, İstanbul \\ (umdurdag@yildiz.edu.tr) ORCID ID 0000 - 0003 - 2049 - 6587 \\ ${ }^{3}$ Yıldız Teknik Üniversitesi, İnşaat Fakültesi, Harita Mühendisliği Bölümü, İstanbul \\ (alihasan@yildiz.edu.tr) ORCID ID 0000 - 0002 - 8490 - 890X \\ ${ }^{4}$ Yıldız Teknik Üniversitesi, İnşaat Fakültesi, Harita Mühendisliği Bölümü, İstanbul \\ (tocalan@yildiz.edu.tr) ORCID ID 0000 - 0003 - 0861 - 013X
}

$\ddot{O} \mathbf{z}$

Bir datumdan diğer bir datuma olan koordinat dönüşümü jeodezinin temel problemlerinden bir tanesidir. Genel olarak problem, bir sistemde tanımlı koordinatların, matematiksel eşitlikler kullanılarak farklı bir başlangıç noktasına sahip sisteme dönüşümüdür. Dönüşüm parametrelerinin hesaplanması için, her iki sistemde koordinatları bilinen yeterli sayıdaki ortak noktanın olması gerekmektedir. Problem iki boyutlu (2B) ya da üç boyutlu (3B) koordinat sistemlerinin dönüşümünü içerir. Koordinat dönüşümünde yaygın olarak kullanılan yöntem, Helmert Dönüşümü olarak da adlandırılan En Küçük Kareler (EKK) yaklaşımıdır. Son yıllarda, Toplam En Küçük Kareler (TEKK) olarak adlandırılan yeni bir yaklaşım, deformasyon analizi, koordinat dönüşümü vb. gibi jeodezik çalışmalarda kullanılmaya başlanmıştır. EKK yaklaşımda, sadece ölçü vektörü hatalı kabul edilirken, TEKK yaklaşımında ölçü vektörünün yanında katsayılar matrisi de hatalı kabul edilmektedir. $\mathrm{Bu}$ çalışmanın amacı, farklı dönüşüm parametreleri kullanarak bu iki yöntemin koordinat dönüşümü problemlerinde performanslarını karşılaştırmak ve $2 \mathrm{~B}$ ağlarda değişen her bir parametrenin etkisini araştırmaktır. Bu amaçla, jeodezik bir ağ yapay olarak üretilmiş ve farklı senaryolarda dönüşüm parametreleri hesaplanmıştır. Yöntemlerin performanslarına ait karşılaştırmalar, kestirilen dönüşüm parametrelerinin norm değerleri dikkate alınarak yapılmıştır. Yöntemleri karşılaştırmak için, dönüşüm parametrelerinin ortalamalarına ait norm değerleri 10000 farklı durum için hesaplanmıştır. Elde edilen sonuçlar, TEKK yaklaşımının hesaplanan norm değerlerine göre daha güvenilir sonuçlar verdiğini göstermiştir.

Anahtar Kelimeler: Toplam En Küçük Kareler (TEKK), En Küçük Kareler (EKK), Koordinat Dönüşümü, Dönüşüm Parametreleri, Norm

\section{Performance of the Least Squares and Total Least Squares Methods on Two Dimensional Coordinate Transformation}

\begin{abstract}
Coordinate transformation from one datum to another is one of the basic problems in geodesy. Generally, the problem is to transform the coordinates defined in a coordinate system into a coordinate system with defined another origin by using mathematical equations. To compute the transformation parameters between two coordinate systems, sufficient number of coordinates of the common points should be known in both systems. The problem involves either two dimensional (2D) or three dimensional (3D) coordinate systems.
\end{abstract}

\footnotetext{
* Sorumlu Yazar
} 
Traditionally, common model used for coordinate transformation is the Least Squares (LS) method known as Helmert Transformation. Recently, a new approach so called Total Least Squares (TLS) has become used in application areas in geodetic studies, such as deformation analysis, coordinate transformation etc. Although only the observation vector is assumed as erroneous according to LS approach, design matrix besides observation vector is assumed as erroneous in TLS approach. The aims of this study were to compare the performance of these two methods in coordinate transformation problems in terms of altering transformation parameters and to investigate the effects of each parameters change to the model in $2 \mathrm{D}$ networks. For these aims, the geodetic networks were simulated and transformation parameters were computed under different scenarios. The comparisons of performance of the methods are considered with the norm of the estimated translation parameters. To compare the methods, the norm criteria of mean of transformation parameters were computed for 10000 different cases. The results showed that TLS estimates more reliable solutions in terms of norm values.

Keywords: Total Least Squares (TLS) Method, Least Square (LS), Coordinate Transformation, Transformation Parameter, Norm

\section{GİRIŞ̧}

Koordinat dönüşümü, jeodezinin en yaygın konularından birisidir. Klasik anlamda iki boyutlu koordinat dönüşümü, öteleme elemanları, ölçek faktörü ve dönme açısından oluşan dönüşüm parametreleri yardımıyla bir datumdan diğer bir datuma koordinatların aktarılması için kullanılır. Mühendislik ölçmelerinde uygulama alanlarındaki artış ve farklı datumlara sahip paftaların birleştirilmesi gibi nedenlerden dolay1, hassas datum dönüşümünün gerekliliğgi de artmıştır. Datum dönüşümündeki problem, iki farklı datumda bulunan koordinatları bilinen ortak noktaları kullanarak dönüşüm parametrelerinin hesaplanması olarak tanımlanabilir.

Literatürde yapılan pek çok çalışmada, dönüşüm parametrelerinin kestirimi için farklı stratejiler kullanılmıştır. Bunların arasında, 2 boyutlu ağlarda Helmert Dönüşümü en yaygın uygulanan yöntemdir (Guobin vd., 2018). Helmert Dönüşümünde dönüşüm elemanları, iki eksen boyunca alınan iki adet öteleme elemanı, bir ölçek faktörü ve iki koordinat sisteminin eksenleri arasındaki bir dönüklük açısıdır. Helmert Dönüşümünde, her iki datumda ortak olacak şekilde koordinatları bilinen en az iki noktaya ihtiyaç vardır. İki nokta, çözüm için gerekli olan minimum ortak nokta sayısını ifade etmektedir. Helmert Dönüşümü, bu ortak noktaların koordinatlarını kullanarak En Küçük Kareler (EKK) prensibine dayalı olarak kestirim yapar.

EKK yaklaşımında, sadece ölçü vektörü hatalı kabul edilir. Ancak, koordinat dönüşümünde olduğu gibi bazı durumlarda, katsayılar matrisini oluşturan parametreler de hatalı olabilir. $\mathrm{Bu}$ nedenle, bu gibi durumlarda, katsayılar matrisinin de hatalı kabul edildiği Toplam En Küçük Kareler (TEKK) yaklaşımı kullanılmalıdır (Golub ve Van Loan, 1980; Van Huffel ve Vandewalle, 1991; Felus, 2004; Schaffrin vd., 2006; Markovsky ve Van Huffel, 2007; Akyılmaz, 2007; Akyılmaz vd., 2007; Schaffrin ve Wieser, 2008). TEKK, katsayılar matrisinin de rasgele hata içerdiği (Errors-in-Variables, EIV) dengeleme modelini önermektedir. Farklı koordinat sistemlerindeki noktaların koordinatlar1, dönüşüm eşitliklerindeki katsayılar matrisinde kendi hata bileşenleri ile değerlendirilmektedir.

Jeodezinin en yaygin olarak kullanılan ve araştırılan konularından birisi olan koordinat dönüşümü, güncelliğini ve uygulama alanlarını koruyan bir problemdir. Dönüşüme etki eden her bir parametrenin araştırılması ve kullanılan yaklaşımların performans değerlendirmeleri ise elde edilen sonuçların güvenilirliği için önemlidir.

\section{YÖNTEM}

\section{1. İki Boyutlu EKK Yaklaşımı (Helmert Dönüşümü)}

Sadece bir sisteme ait stokastik modelin hata içerdiğini kabul eden ve günümüzde Helmert Dönüşümü olarak adlandırılan 2 boyutlu koordinat dönüşümü, F.R. Helmert tarafından formüle edilmiştir. Bu yaklaşımda, dönüşüm parametreleri EKK yaklaşımı ile kestirilmektedir. 2 Boyutlu Helmert dönüşüm problemi, iki öteleme elemanı, bir dönüklük bileşeni ve bir ölçek faktörü olmak üzere dört 
adet dönüşüm parametresini içermektedir. Parametrelerin kestirimi için, iki farklı sistemde ortak olan noktalar kullanılır. İki boyutlu Helmert Dönüşüm problemine ait eşitlikler, (1) ve (2)' de verilmiştir.

$$
\mathrm{x}=\mathrm{t}_{\chi}+\mathrm{k} \cos \varepsilon \chi-\mathrm{k} \sin \varepsilon \gamma
$$

$$
\mathrm{y}=\mathrm{t}_{\gamma}+\mathrm{k} \sin \varepsilon \chi+\mathrm{k} \cos \varepsilon \gamma
$$

Burada $\chi$ ve $\gamma$ birinci sistemdeki koordinatları, $\mathrm{x}$ ve $\mathrm{y}$ ikinci sistemdeki koordinatları, $\mathrm{t}_{\chi}$ ve $\mathrm{t}_{\gamma}$ öteleme elemanlarını, k ölçek faktörünü, $\varepsilon$ dönüklük bileşenini ifade etmektedir. A katsayılar matrisinin elemanları aşağıdaki gibi yazılır,

$\mathbf{A}_{\mathbf{i}}=\left[\begin{array}{rrrr}1 & 0 & \chi_{i} & -\gamma_{i} \\ 0 & 1 & \gamma_{i} & \chi_{i}\end{array}\right] \mathrm{i}=1, \ldots \ldots, \mathrm{n}$

Burada, $\mathrm{n}$ her iki sistemde ortak olan nokta sayısıdır. $\mathbf{l}=\left[\begin{array}{ll}x & y\end{array}\right]^{T}$ dönüşüm problemindeki ölçü vektörünü göstermektedir. Ölçü vektörü ikinci sistemdeki tüm eşlenik noktaların koordinatlarını içerir. Dönüşüm parametrelerini $\quad \boldsymbol{\beta}=$ $\left[\begin{array}{llll}t_{\chi} & t_{\gamma} & k \cos \varepsilon & k \sin \varepsilon\end{array}\right]^{T}$ kestirmek için, tek bir ortak nokta için düzeltme denklemleri aşağıdaki gibi oluşturulur:

$$
\mathbf{v}_{\mathbf{L S}}=\mathbf{A} \boldsymbol{\beta}-\mathbf{l}
$$

(4)

$$
\left[\begin{array}{l}
v_{\chi} \\
v_{\gamma}
\end{array}\right]=\left[\begin{array}{cccc}
1 & 0 & \chi & -\gamma \\
0 & 1 & \gamma & \chi
\end{array}\right]\left[\begin{array}{c}
t_{\chi} \\
t_{\gamma} \\
k \cos \varepsilon \\
k \sin \varepsilon
\end{array}\right]-\left[\begin{array}{l}
x \\
y
\end{array}\right]
$$

Ortak noktalar yardımı ile iki sistem arasındaki dönüşüm parametreleri kestirildikten sonra $\chi$ ve $\gamma$ koordinatları, ikinci sistemde $\mathrm{x}$ ve $\mathrm{y}$ olarak adlandırılan nokta koordinatlarına dönüştürülürler (Şekil 1).

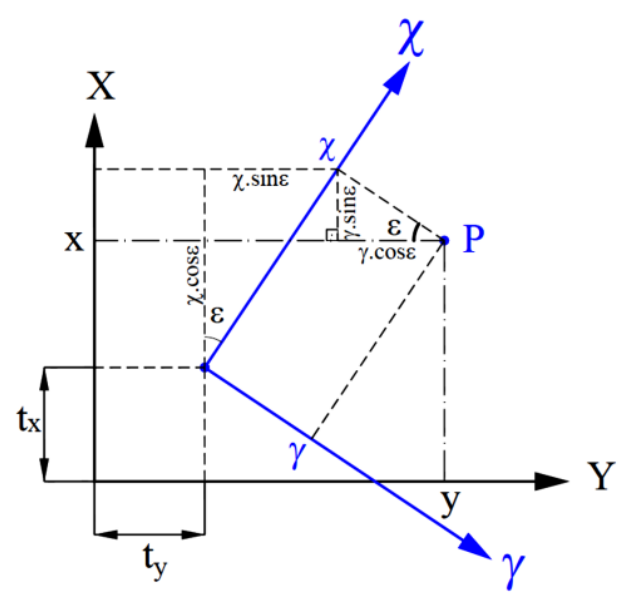

Şekil 1. İki Boyutlu Koordinat Dönüşümü

\subsection{Toplam En Küçük Kareler (TEKK)}

Golub ve Van Loan (1980) tarafindan ortaya atılan TEKK yöntemi, ölçü vektörü ve katsayılar matrisinin her ikisinin de rasgele hata (aynı varyans değerine sahip ve sifır ortalamalı) içerdiği EIV dengeleme modeli olarak tanımlanmaktadır. TEKK yaklaşımındaki fonksiyonel model, genel haliyle aşağıda verildiği gibi yazılabilir,

$\mathbf{l}+\mathbf{e}=(\mathbf{A}-\mathbf{E}) \boldsymbol{\beta} ; \mathbf{l}+\mathbf{e}=\tilde{\mathbf{l}} ; \mathbf{A}-\mathbf{E}=\widetilde{\mathbf{A}}$

(6)

Burada, $\mathbf{A}(m \times n)$ boyutlarında $(\operatorname{rank}(\mathbf{A})=$ $m<n)$ olan katsayılar matrisini; $\mathbf{l}(m \times 1)$ boyutundaki ölçü vektörünü; e, l vektörüne ait hata vektörünü; $\mathbf{E} \mathbf{A}$ matrisine ait hata matrisini; $\quad \boldsymbol{\beta} \quad(m \times 1) \quad$ boyutundaki bilinmeyenler vektörünü ifade etmektedir.

$\min _{[\mathbf{e} ; \mathbf{E}]}\|[\mathbf{E} ; \mathbf{e}]\|_{F} \rightarrow \mathbf{l}+\mathbf{e}=(\mathbf{A}-\mathbf{E}) \boldsymbol{\beta}$

Burada, $\quad\|\mathbf{H}\|_{F} \quad n \times m$ boyutundaki $\quad \mathrm{H}$ matrisinin Frobenius normunu, [E; e] $n \times$ $(m+1)$ boyutlu her iki sistemdeki hata matrisini ifade eder ve aşağıda verildiği haliyle tanımlanır;

$\|\mathbf{H}\|_{F}=\sqrt{\sum_{i=1}^{n} \sum_{j=1}^{m} h_{i j}^{2}}=\sqrt{\operatorname{tr}\left(\mathbf{H}^{T} \mathbf{H}\right)}$

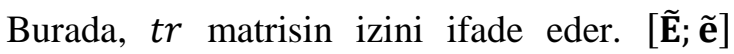
matrisinin minimum değere sahip olmasına dayalı olarak $\boldsymbol{\beta}$ hesaplanır.

$(\mathbf{A}-\tilde{\mathbf{E}}) \boldsymbol{\beta}=\mathbf{1}+\tilde{\mathbf{e}}$

(9) 
Burada; dönüşümde her iki sistemdeki hataları da içeren genişletilmiş $[\mathbf{A} ; \mathbf{l}]$ matrisi; [E; e]' nin Frobenius normunu minimum yapacak şekilde değiştirilir. Bu değişiklik genişletilmiş [A; l] matrisinin sütunları arasında lineer bir ilişki doğurur. Ayrıca, $(m+1)$ rank değerine sahip $[\mathbf{A} ; \mathrm{l}]$ matrisi; $\mathrm{m}$ rank değerine sahip $[\mathbf{A}-\mathbf{E} ; \mathbf{l}+\mathbf{e}]=[\widehat{\mathbf{A}} ; \hat{\mathbf{l}}]$ matrisi ile değiştirilir. $\mathrm{Bu}$ durum, î nin sütunlarının $\widehat{\mathbf{A}}$ 'nın sütunları ile lineer bağımlı olduğu anlamına gelmektedir (Felus, 2004).

Genişletilmiş $[\mathbf{A} ; \mathbf{l}]$ matrisinin tekil değer ayrışımı (TDA) (Singular Value Decomposition - SVD) gerçekleştirilirse;

$[\mathbf{A} ; \mathbf{l}]=\mathbf{U} \mathbf{\Sigma} \mathbf{V}^{T}$

$\mathbf{U}=\left[u_{1}, \ldots, u_{2}\right] \in R^{n \times n} ; \mathbf{V}=\left[v_{1}, \ldots, v_{m+1}\right] \in$ $R^{(m+1) \times(m+1)} ; \quad \boldsymbol{\Sigma}=\operatorname{diag}\left(\sigma_{1}, \cdots, \sigma_{m+1}\right) \quad \in$ $R^{n \times(m+1)}$ şeklinde hesaplanır. Burada $\boldsymbol{\Sigma}$ matrisinin köşegen elemanları tekil değerlere, $\left[\sigma_{i j}=\sigma_{i} ; i=1, \cdots,(m+1) ; i=j\right]$, köşegen dışındaki elemanları da 0 ' a eşit olur $\left[\sigma_{i j}=\right.$ $0 ; i \neq j]$ (Felus, 2004).

\section{Teorem 1}

(10) denkleminde $[\mathbf{A} ; \mathbf{l}]$ matrisinin TDA' sinda $\sigma_{m}>\sigma_{m+1}$ ve $v_{m+1, m+1} \neq 0 \quad$ olduğu varsayılır. $\mathrm{Bu}$ durumda, genişletilmiş $[\mathbf{A} ; \mathbf{l}]$ matrisinin TEKK kestirimi aşağıdaki şekilde gerçekleştirilir:

$$
[\widehat{\mathbf{A}} ; \hat{\mathbf{l}}]=\mathbf{U} \widehat{\boldsymbol{\Sigma}} \mathbf{V}^{\mathbf{T}}, \widehat{\boldsymbol{\Sigma}}=\operatorname{diag}\left(\sigma_{1}, \ldots, \sigma_{m}, 0\right)
$$

$\sigma_{m}>\sigma_{m+1}$ ve $v_{m+1, m+1} \neq 0$ koşulları $\mathbf{A}$ matrisi tam ranka sahip olduğunda genellikle sağlanır. Burada $\hat{\mathbf{l}}=\mathbf{l}+\mathbf{e}$ ve $\widehat{\mathbf{A}}=\mathbf{A}+\mathbf{E}$ şeklinde ifade edilirse (6) nolu denklem aşağıdaki şekilde tekrar yazılabilir.

$$
[\widehat{\mathbf{A}} ; \hat{\mathbf{1}}]\left[\begin{array}{c}
\boldsymbol{\beta} \\
-1
\end{array}\right]=0
$$

$[\boldsymbol{\beta} ;-1]^{T}$ vektörü genişletilmiş $[\widehat{\mathbf{A}} ; \mathbf{1}]$ matrisinin soldan sıfır uzayıdır; TEKK problemi TDA kullanılarak çözülebilir. Bunun yanında, $\mathrm{v}_{m+1}$ ( $\mathbf{V}^{\prime}$ nin son sütunun son bileşeni), -1 değerine sahip oluncaya kadar ölçeklendirilerek $[\boldsymbol{\beta} ;-1]^{T}$ vektörü elde edilir (Felus, 2004).

\section{Teorem 2}

(7) nolu denklemde verilen koşula göre parametreler vektörü aşağıdaki şekilde hesaplanabilir:

$\widehat{\boldsymbol{\beta}}=-\frac{1}{v_{m+1, m+1}}\left[v_{1, m+1}, \ldots, v_{m, m+1}\right]^{T}$

TEKK probleminin tek çözümü; en küçük tekil değer ve $[\mathbf{A} ; \mathbf{l}]$ matrisinin sağ tekil vektörüyle ilişkilendirilir. TEKK düzeltme matrisini $[\Delta \mathbf{A} ; \Delta \mathbf{l}]$ hesaplayabilmek için, Teorem 1 ve (13) nolu denklem birlikte düşünülürse, aşağıdaki denklem elde edilir:

$[\Delta \mathbf{A} ; \Delta \mathbf{l}]=[\mathbf{A} ; \mathbf{l}]-[\widehat{\mathbf{A}} ; \hat{\mathbf{l}}]=\sigma_{m+1} u_{m+1} v_{m+1}^{T}$

Burada, $[\widehat{\boldsymbol{\beta}} ;-1]^{\mathrm{T}}$ vektörü, $[\mathbf{A} ; \mathbf{1}]^{T}[\mathbf{A} ; \mathbf{1}]^{\prime} \operatorname{nin}$ özdeğeri ile ilgili özvektördür. Sonuç olarak, özvektör denklemi aşağıdaki gibi yazılabilir:

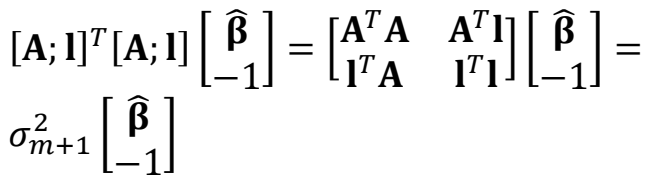

$\widehat{\boldsymbol{\beta}}$ bilinmeyen parametrelerin kestirimi (15) nolu denklemin ilk satırı göz önünde bulundurularak (16) nolu eşitlikte hesaplanabilir.

$\left(\mathbf{A}^{T} \mathbf{A}-\sigma_{m+1}^{2} I\right) \widehat{\boldsymbol{\beta}}=\mathbf{A}^{T} \mathbf{l}$

(16) nolu eşitlik TEKK çözümünün normal denklemleri olarak kabul edilebilir. $\sigma_{m}>$ $\sigma_{m+1}$ ve $v_{m+1, m+1} \neq 0$, olduğu için $\left(\mathbf{A}^{T} \mathbf{A}-\right.$ $\left.\sigma_{m+1}^{2} \mathbf{I}\right)$ matrisi pozitiftir; bu şekilde $\widehat{\boldsymbol{\beta}}$ (17) nolu denklem ile hesaplanır.

$\widehat{\boldsymbol{\beta}}=\left(\mathbf{A}^{T} \mathbf{A}-\sigma_{m+1}^{2} \mathbf{I}\right)^{-1} \mathbf{A}^{T} \mathbf{l}$

TEKK yönteminde katsayllar matrisinin sabit sütunlarl

TEKK yönteminde hem ölçülerin hem de katsayılar matrisinin (A) hatalı olarak düşünülmesine karşın; TEKK çözümlerinden sonra bazı değerler değişmez, sabit kalır. Bu duruma koordinat dönüşüm problemlerinde öteleme parametrelerinin katsayıları örnek verilebilir. Bu durumda A katsayılar matrisi ve $\boldsymbol{\beta}$ bilinmeyen vektörü alt kısımlara ayrılır (Akyılmaz, 2007).

$\mathbf{A}=\left[\mathbf{A}_{1} ; \mathbf{A}_{2}\right]$; 
$\mathbf{A}_{\mathbf{1}} \in R^{n \times m_{1}} \mathrm{ve}_{\mathbf{2}} \in R^{n \times m_{2}}$

(18)

$\boldsymbol{\beta}=\left[\boldsymbol{\beta}_{1}^{T} ; \boldsymbol{\beta}_{2}^{T}\right]^{T} ;$

$\boldsymbol{\beta}_{1} \in R^{m_{1} \times 1} \mathrm{ve} \boldsymbol{\beta}_{2} \in R^{m_{2} \times 1}$

(19)

Burada $\mathbf{A}_{\mathbf{1}}, \mathbf{A}_{\mathbf{2}}, \boldsymbol{\beta}_{\mathbf{1}}, \boldsymbol{\beta}_{\mathbf{2}}$ sirasiyla A katsayılar matrisinin ve $\boldsymbol{\beta}$ bilinmeyen parametre vektörünün alt kısımlara bölünmüş halidir. $\mathbf{A}_{\mathbf{1}}$ matrisinin sütunlarının bilindiği kabul edilirse; TEKK problemi aşağıdaki şekilde oluşturulabilir:

$\min _{[\widehat{A} ; 1]}\left\|\left[\mathbf{A}_{2} ; \mathbf{l}\right]-\left[\widehat{\mathbf{A}}_{2} ; \hat{\mathbf{l}}\right]\right\|_{F} \rightarrow \widehat{\boldsymbol{A}} \widehat{\boldsymbol{\beta}}=$ $\left[\mathbf{A}_{1} ; \widehat{\mathbf{A}}_{2}\right]\left[\begin{array}{l}\widehat{\boldsymbol{\beta}}_{1} \\ \widehat{\boldsymbol{\beta}}_{2}\end{array}\right]=\hat{\mathbf{l}}$

$\mathrm{Bu}$ problemin çözülebilmesi için bilinen sütunlarla diğerlerini ayırmak gerekir. $\mathrm{Bu}$ işlem QR çarpanlarına ayırma yöntemi ile gerçekleştirilebilir. QR çarpanlarına ayırma yöntemi dörtgen matrisleri bir ortogonal matris çarpımı $\mathbf{Q}$ ve bir de üst üçgen matris $\mathbf{R}$ olarak ifade etmemizi sağlar (Felus, 2004).

Genişletilmiş matrisin QR çarpanlara ayrılması aşağıdaki şekilde gerçekleştirilir.

$$
[\mathbf{A} ; \mathbf{l}]=\mathbf{Q R}
$$

$\mathbf{Q}^{\mathrm{T}}\left[\mathbf{A}_{1} ; \mathbf{A}_{2} ; \mathbf{l}\right]=\left[\begin{array}{ccc}\mathbf{R}_{11} & \mathbf{R}_{12} & \mathbf{R}_{1 \mathrm{~b}} \\ \mathbf{0} & \mathbf{R}_{22} & \mathbf{R}_{2 b}\end{array}\right]$

Ortogonal Q matrisinin sütunlarının öklit formu 1' e ve Frobenius normu $\sqrt{n}$, ' ye eşittir. Ayrica, $\mathbf{R}$ matrisi $\mathbf{R}_{\mathbf{1 1}}, \mathbf{R}_{\mathbf{1 2}}, \mathbf{R}_{\mathbf{2 2}}, \mathbf{R}_{\mathbf{1 b}}$ ve $\mathbf{R}_{\mathbf{2 b}}$ şeklinde alt matrislere ayrilabilir. $\mathrm{Bu}$ matrislerin boyutları sirasiyla $\left(m_{1} \times m_{1}\right)$, $\left(m_{1} \times m-m_{1}\right),\left(n-m_{1} \times m-m_{1}\right),\left(m_{1} \times\right.$ $1),\left(n-m_{1} \times 1\right)$ şeklindedir.

Felus (2004)' te verildiği şekilde karma EKKTEKK probleminin çözümü iki adımdan oluşmaktadır:

1. $\widehat{\boldsymbol{\beta}}_{2}$ parametre vektörü indirgenmiş sistem için Teorem 2 kullanılarak hesaplanır:

$\mathbf{R}_{\mathbf{2 2}} \boldsymbol{\beta}_{\mathbf{2}} \approx \mathbf{R}_{\mathbf{2 b}}$

(23)

$\widehat{\mathbf{R}}_{\mathbf{2 2}} \quad$ ve $\quad \widehat{\mathbf{R}}_{\mathbf{2 b}} \quad$ Teorem 1 kullanılarak hesaplanabilir.
2. $\widehat{\boldsymbol{\beta}}_{1}$ parametre vektörü aşağıdaki denklemlerde yerine koyma tekniği kullanılarak hesaplanabilir.

$\mathbf{R}_{\mathbf{1 1}} \widehat{\boldsymbol{\beta}}_{\mathbf{1}}=\mathbf{R}_{\mathbf{1 b}}-\mathbf{R}_{\mathbf{1 2}} \widehat{\boldsymbol{\beta}}_{\mathbf{2}}$

(24)

Buraya kadar anlatılan yöntemler tüm değerlere ait varyans değerlerinin aynı kabul edildiği klasik TEKK çözümünü içermektedir. Buna karşın, katsayılar matrisinin ve ölçülerin varyans değerleri farklı olabilir. $\mathrm{Bu}$ tür problemlerin çözümünde Genelleștirilmiş TEKK (GTEKK) yöntemi uygulanır (Felus, 2004; Neitzel, 2010).

GTEKK yönteminde D ve $\mathbf{C}$ olarak iki köşegen ağırlık matrisi tanımlanır. $\mathbf{D}, n \times n$ boyutunda ölçülerin ağırlık matrisini ifade etmektedir $\left(\mathbf{D}=\operatorname{diag}\left(\mathbf{d}_{\mathbf{1}}, \ldots, \mathbf{d}_{\mathbf{n}}\right)\right)$. Ayrica $\mathbf{C}$, $(\mathrm{m}+1) \times(\mathrm{m}+1)$ boyutunda $\mathbf{A}_{2}$ sütunundaki katsayılar matrisine ilișkin ağırlık matrisini ifade etmektedir $\left(\mathbf{C}=\operatorname{diag}\left(\mathbf{c}_{\mathbf{1}}, \ldots, \mathbf{c}_{\mathbf{m}+\mathbf{1}}\right)\right) . \mathrm{Bu}$ tanımlara göre GTEKK probleminin çözümü aşağıdaki şekilde verilebilir:

$$
\begin{aligned}
& \min _{\left[\mathrm{e} ; E_{A 2}\right]}\left\|\mathbf{D}\left[\boldsymbol{E}_{\boldsymbol{A 2}} ; \mathrm{e}\right] \mathbf{C}\right\|_{F} \rightarrow \mathbf{b}+\mathbf{e}= \\
& \left(\mathbf{A}_{1} ; \mathbf{A}_{2}+\mathbf{E}_{\boldsymbol{A} 2}\right)\left[\begin{array}{l}
\widehat{\boldsymbol{\beta}}_{1} \\
\widehat{\boldsymbol{\beta}}_{2}
\end{array}\right]
\end{aligned}
$$

Felus (2004) GTEKK probleminin çözümünü 3 adımda ortaya koymaktadır:

1. Genişletilmiş $\mathbf{D}[\mathbf{A} ; \mathbf{l}]$ matrisi $Q R$ çarpanlarına ayrılır.

$$
\mathbf{Q}^{\mathbf{T}} \mathbf{D}\left[\mathbf{A}_{1} ; \mathbf{A}_{2} ; \mathbf{l}\right]=\left[\begin{array}{ccc}
\mathbf{R}_{11} & \mathbf{R}_{12} & \mathbf{R}_{1 b} \\
\mathbf{0} & \mathbf{R}_{22} & \mathbf{R}_{2 b}
\end{array}\right]
$$

2. İndirgenmiş sistem için $\widehat{\boldsymbol{\beta}}_{2}$ parametre vektörü klasik TEKK yöntemine göre hesaplanır.

$\left[\mathbf{R}_{22} ; \mathbf{R}_{2 b}\right] \mathbf{C}\left(\mathbf{C}^{-1}\left[\begin{array}{c}\boldsymbol{\beta}_{2} \\ -1\end{array}\right]\right) \approx \mathbf{0}$

(27) nolu eşitliği çözümü için $\left[\mathbf{R}_{\mathbf{2 2}} ; \mathbf{R}_{\mathbf{2 b}}\right] \mathbf{C}=$ $\mathbf{U} \boldsymbol{\Sigma} \mathbf{V}^{\mathbf{T}}$ eşitliğinin TDA' s1 hesaplanır.

$\widehat{\boldsymbol{\beta}}=-\frac{1}{\mathbf{c}_{m+1} \mathbf{v}_{m+1, m+1}} \mathbf{C}_{1 \ldots m}\left[v_{1, m+1}, \ldots, v_{m, m+1}\right]^{T}$

Burada, $\mathbf{C}_{1 \ldots m}=\operatorname{diag}\left(c_{1}, \ldots, c_{m}\right)$ 
3. $\widehat{\boldsymbol{\beta}}_{\mathbf{1}}$ parametresi, ikinci adımda hesaplanan $\widehat{\boldsymbol{\beta}}_{2}$ parametresinin yardımıyla aşağıdaki gibi hesaplanır.

$$
\mathbf{R}_{\mathbf{1 1}} \boldsymbol{\beta}_{\mathbf{1}}=\mathbf{R}_{\mathbf{1 b}}-\mathbf{R}_{\mathbf{1 2}} \widehat{\boldsymbol{\beta}}_{\mathbf{2}}
$$

\section{BULGULAR}

Son yıllarda koordinat dönüşümü ile ilgi pek çok çalışma yapılmasına karşın, TEKK yöntemi kullanılarak koordinat dönüşümünün güvenirliği ile ilgili çalışma yapılmamıştır. Her iki yöntem, doğrultu-kenar ölçülerine dayalı olarak yapay olarak oluşturulmuş ağlarda (Şekil 2) test edilmiştir. Kenar uzunlukları 212 $\mathrm{m}$ ile $370 \mathrm{~m}$ aralığında değişen 9 noktalı test ağında, kullanılan alet doğruluğu kenar ölçümünde $\pm(3+2 p p m)$ ve doğrultu ölçümünde $\pm 2^{c c}$ olarak kabul edilmiştir. Yöntemlerin performanslarına ait karşılaştırmalar, kestirilen dönüşüm parametrelerinin norm değerleri dikkate alınarak yapılmıştır. Norm değeri, analiz yönteminin performans ölçümünde kullanılan bir değerdir (Hekimoglu ve Erenoglu 2013). Çalışmada, birinci ve ikinci datumlar $100 \times$ 100 kez üretilmiştir. Burada, ikinci datum, Tablo 1, 2, 3 ve 4' te verilen durumlar göz önüne alınarak orijinal dönüşüm parametreleri kullanılarak üretilmiştir. Simülasyon, 10000 kez çalıştırılmış ve dönüşüm parametreleri 10000 tekrar için hesaplanmıştır. Norm değerleri, orijinal dönüşüm parametreleri ile kestirilen değerlerin arasındaki farkların mutlak değerlerine ait ortalama değerden hesaplanmıştır (Hekimoglu ve Erenoglu, 2013).

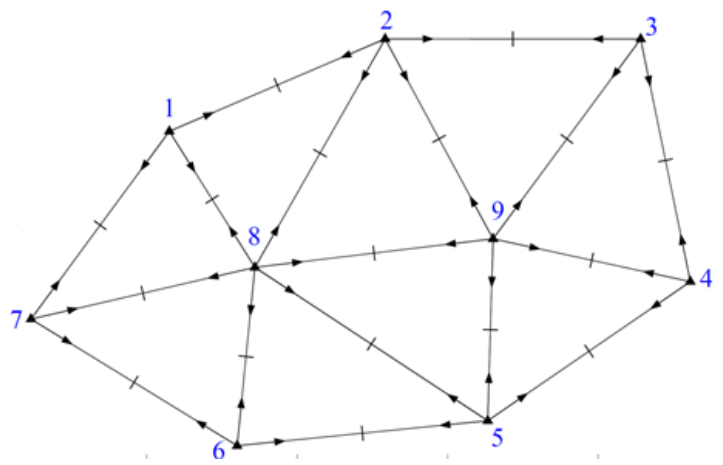

Şekil 2. 2B yapay olarak üretilmiş ağ (Doğrultu-Kenar)

2 Boyutlu ağda, dört adet dönüşüm parametresi (iki öteleme, bir dönüklük ve bir ölçek faktörü) sırasıyla $t_{x}, t_{y}, \varepsilon$ ve $k$ olarak gösterilmiştir (Tablo 1-4). Her durumda, ölçek faktörü sabit ("1") olarak alınmıştır. Tablolarda farklı senaryolara ait 2 Boyutlu ağ çözümleri verilmiştir. Farklı senaryolardaki durumlar, öteleme elemanları ile dönüklük bileşeni arasındaki ilişkiyi ortaya koymak için oluşturulmuş ve bu sayede EKK ile TEKK çözümlerinin güvenilirliği araştırılmıştır.

Tablo 1' de, dönüklük bileşeni $0^{\circ}$ ile $10^{\circ}$ arasında arttırılırken, öteleme elemanları değiştirilmeyerek $10 \quad \mathrm{~cm}$ gibi küçük değişimlerdeki durum dikkate alınmıştır. Helmert dönüşümünden elde edilen sonuçlar incelendiğinde, parametrelere ait norm değerlerinin 2.3556 ve 2.3598 arasında birbirine yakın değerlerde değiştiği ve anlamlı bir farkın olmadığ 1 görülmüştür. Ancak, dönüklük açısının $10^{\circ}$ olduğu durumda norm değerlerinde farklılıklar bulunmuştur. Dönüklük açısı $10^{\circ}$ olduğunda hesaplanan norm değeri diğer durumlardan daha küçük sonuç vermiştir. Bu durum, EKK yaklaşımının 2 Boyutlu ağlarda dönüklük açısının büyük olduğu durumlar için güvenilir sonuçlar verdiğini göstermektedir. Küçük dönüklük açıları için daha az hassas sonuçlara ulaşılmıştır.

Benzer sonuçlar, TEKK yaklaşımı ile elde edilen çözümlerde de görülmüştür. Parametrelere ait norm değerleri 1.2128 ve 1.2145 arasında değişmektedir. Dönüklük açısının $10^{\circ}$ olduğu durumda ise en küçük norm değerleri elde edilmiştir.

EKK ve TEKK çözümleri karşılaştırıldığında, TEKK yaklaşımı ile elde edilen norm değerinin EKK çözümünden elde edilen değerden iki kat daha küçük olduğu görülmektedir. Tablo 2 ve 3 ' te, dönüklük bileşeni $0^{\circ 1}$ dan $10^{\circ 1}$ ye çıarılırken, $\mathrm{x}$ ve $\mathrm{y}$ yönlerindeki öteleme elemanları aynı anda 1 m' den 100 m' ye arttırılmıştır. Bu durumda, her bir senaryo için EKK ve TEKK sonuçları incelendiğinde, Tablo $1^{\prime}$ de elde edilen sonuçlara benzer olarak dönüklük açısının $10^{\circ}$ olduğu durumda norm değerlerinde değişmenin olduğu görülmektedir. Tablo 4 ' te verildiği gibi öteleme elemanları $1000 \mathrm{~m}$ olarak alınmış ve son senaryodaki durum değerlendirilmiştir. Burada, en yüksek öteleme değerleri verilmiş olmasına rağmen diğer 
durumlarda elde edilen sonuçlarla benzer sonuçlar elde edilmiştir.

Tablo 1. $t_{x}=t_{y}=10 \mathrm{~cm}$ için $2 \mathrm{~B}$ ağda elde edilen sonuçlar

\begin{tabular}{lllll}
\hline Senaryolar & EKK & & TEKK & \\
\hline \multirow{4}{*}{$t_{x}(\mathrm{~m})$} & Ortalama & Norm & Ortalama & Norm \\
\cline { 2 - 5 }$t_{y}(\mathrm{~m})$ & $\hat{t}_{x}(\mathrm{~m})$ & & $\hat{t}_{x}(\mathrm{~m})$ & \\
$k$ & $\hat{t}_{y}(\mathrm{~m})$ & & $\hat{t}_{y}(\mathrm{~m})$ & \\
$\varepsilon\left({ }^{\circ}\right)$ & $\hat{k}$ & & $\hat{k}$ & \\
\hline $10 \mathrm{~cm}$ & $\hat{\varepsilon}\left({ }^{\circ}\right)$ & & $\hat{\varepsilon}\left({ }^{\circ}\right)$ & \\
$10 \mathrm{~cm}$ & 0.5071 & 2.3556 & 0.3817 & 1.2128 \\
1 & -2.2201 & & -1.0796 & \\
$0^{\prime \prime}$ & 0.9999 & & 0.9999 & \\
\hline $10 \mathrm{~cm}$ & 0.0004 & & 0.0004 & \\
$10 \mathrm{~cm}$ & 0.5072 & 2.3556 & 0.3818 & 1.2128 \\
1 & -2.2202 & & -1.0796 & \\
$0.00278^{\circ}\left(10^{\prime \prime}\right)$ & 0.9999 & & 0.9999 & \\
\hline $10 \mathrm{~cm}$ & 0.0028 & & 0.0028 & \\
$10 \mathrm{~cm}$ & 0.5142 & 2.3598 & 0.3919 & 1.2145 \\
1 & -2.2231 & & -1.0789 & \\
$0.16667^{\circ}\left(10^{\prime}\right)$ & 0.9999 & & 0.9999 & \\
\hline $10 \mathrm{~cm}$ & 0.1667 & & 0.1667 & \\
$10 \mathrm{~cm}$ & 0.8056 & $\mathbf{1 . 9 7 9 9}$ & 1.0297 & $\mathbf{1 . 0 8 0 8}$ \\
1 & -1.7499 & & -0.4511 & \\
$10^{\circ}$ & 0.9999 & & 0.9999 & \\
\hline & 10.0000 & & 10.0000 & \\
\hline
\end{tabular}

Tablo 2. $t_{x}=t_{y}=1 \mathrm{~m}$ için $2 \mathrm{~B}$ ağda elde edilen sonuçlar

\begin{tabular}{lllll}
\hline Senaryolar & EKK & & TEKK & \\
\hline \multirow{2}{*}{$t_{x}(\mathrm{~m})$} & Ortalama & Norm & Ortalama & Norm \\
\cline { 2 - 5 }$t_{y}(\mathrm{~m})$ & $\hat{t}_{x}(\mathrm{~m})$ & & $\hat{t}_{x}(\mathrm{~m})$ & \\
$k$ & $\hat{t}_{y}(\mathrm{~m})$ & & $\hat{t}_{y}(\mathrm{~m})$ & \\
$\varepsilon\left(^{\circ}\right)$ & $\hat{k}$ & & $\hat{k}$ & \\
\hline $1 \mathrm{~m}$ & $\hat{\varepsilon}\left(^{\circ}\right)$ & & $\hat{\varepsilon}\left(^{\circ}\right)$ & \\
$1 \mathrm{~m}$ & 1.4071 & 2.3556 & 1.2817 & 1.2128 \\
1 & -1.3201 & & -0.1796 & \\
$0^{\prime \prime}$ & 0.9999 & & 0.9999 & \\
\hline $1 \mathrm{~m}$ & 0.0004 & & 0.0004 & \\
$1 \mathrm{~m}$ & 1.4072 & 2.3556 & 1.2818 & 1.2129 \\
1 & -1.3202 & & -0.1796 & \\
$0.00278^{\circ}\left(10^{\prime \prime}\right)$ & 0.9999 & & 0.9999 & \\
\hline $1 \mathrm{~m}$ & 0.0028 & & 0.0028 & \\
$1 \mathrm{~m}$ & 1.4142 & 2.3598 & 1.2919 & 1.2145 \\
1 & -1.3231 & & -0.1789 & \\
$0.16667^{\circ}\left(10^{\prime}\right)$ & 0.9999 & & 0.9999 & \\
\hline $1 \mathrm{~m}$ & 0.1667 & & 0.1667 & \\
$1 \mathrm{~m}$ & 1.7056 & $\mathbf{1 . 9 7 9 9}$ & 1.9297 & $\mathbf{1 . 0 8 0 8}$ \\
1 & -0.8499 & & 0.4489 & \\
$10^{\circ}$ & 0.9999 & & 0.9999 & \\
\hline & 10.0000 & & 10.0000 & \\
\hline
\end{tabular}

Tablo 3. $t_{x}=t_{y}=100 \mathrm{~m}$ için $2 \mathrm{~B}$ ağda elde edilen sonuçlar

\begin{tabular}{lllll}
\hline Senaryolar & EKK & & TEKK & \\
\hline \multirow{3}{*}{$t_{x}(\mathrm{~m})$} & Ortalama & Norm & Ortalama & Norm \\
\cline { 2 - 5 }$t_{y}(\mathrm{~m})$ & $\hat{t}_{x}(\mathrm{~m})$ & & $\hat{t}_{x}(\mathrm{~m})$ & \\
$k$ & $\hat{t}_{y}(\mathrm{~m})$ & & $\hat{t}_{y}(\mathrm{~m})$ & \\
$\varepsilon\left(^{\circ}\right)$ & $\hat{k}$ & & $\hat{k}$ & \\
\hline 100 & $\hat{\varepsilon}\left(^{\circ}\right)$ & & $\hat{\varepsilon}\left(^{\circ}\right)$ & \\
100 & 100.4071 & 2.3556 & 100.2816 & 1.2128 \\
1 & 97.6799 & & 98.8204 & \\
$0^{\prime \prime}$ & 0.9999 & & 0.9999 & \\
\hline 100 & 0.0004 & & 0.0004 & \\
& 100.4072 & 2.3556 & 100.2818 & 1.2128
\end{tabular}

\begin{tabular}{lllll}
100 & 97.6798 & & 98.8204 & \\
1 & 0.9999 & & 0.9999 & \\
$0.00278^{\circ}\left(10^{\prime \prime}\right)$ & 0.0028 & & 0.0028 & \\
\hline 100 & 100.4142 & 2.3598 & 100.2919 & 1.2145 \\
100 & 97.6769 & & 98.8211 & \\
1 & 0.9999 & & 0.9999 & \\
$0.16667^{\circ}\left(10^{\prime}\right)$ & 0.1667 & & 0.1667 & \\
\hline 100 & 100.7056 & $\mathbf{1 . 9 7 9 9}$ & 100.9297 & $\mathbf{1 . 0 8 0 7}$ \\
100 & 98.1501 & & 99.4489 & \\
1 & 0.9999 & & 0.9999 & \\
$10^{\circ}$ & 10.0000 & & 10.0000 & \\
\hline
\end{tabular}

Tablo 4. $t_{x}=t_{y}=1000 \mathrm{~m}$ için $2 \mathrm{~B}$ ağda elde edilen sonuçlar

\begin{tabular}{lllll}
\hline Senaryolar & EKK & & TEKK & \\
\hline \multirow{2}{*}{$t_{x}(\mathrm{~m})$} & Ortalama & Norm & Ortalama & Norm \\
\cline { 2 - 5 }$t_{y}(\mathrm{~m})$ & $\hat{t}_{x}(\mathrm{~m})$ & & $\hat{t}_{x}(\mathrm{~m})$ & \\
$k$ & $\hat{t}_{y}(\mathrm{~m})$ & & $\hat{t}_{y}(\mathrm{~m})$ & \\
$\varepsilon\left(^{\circ}\right)$ & $\hat{k}$ & & $\hat{k}$ & \\
\hline 1000 & $\hat{\varepsilon}\left(^{\circ}\right)$ & & $\hat{\varepsilon}\left(^{\circ}\right)$ & \\
1000 & 1000.4071 & 2.3556 & 1000.2816 & 1.2128 \\
1 & 997.6799 & & 998.8203 & \\
$0^{\prime \prime}$ & 0.9999 & & 0.9999 & \\
\hline 1000 & 0.0004 & & 0.0004 & \\
1000 & 1000.4072 & 2.3556 & 1000.2818 & 1.2128 \\
1 & 997.6798 & & 998.8204 & \\
$0.00278^{\circ}\left(10^{\prime \prime}\right)$ & 0.9999 & & 0.9999 & \\
\hline 1000 & 10028 & & 0.0028 & \\
1000 & 997.6769 & & 998.8211 & \\
1 & 0.9999 & & 0.9999 & \\
$0.16667^{\circ}\left(10^{\prime}\right)$ & 0.1667 & & 0.1667 & \\
\hline 1000 & 1000.7056 & $\mathbf{1 . 9 7 9 9}$ & 1000.9297 & $\mathbf{1 . 0 8 0 8}$ \\
1000 & 998.1501 & & 999.4489 & \\
1 & 0.9999 & & 0.9999 & \\
$10^{\circ}$ & 10.0000 & & 10.0000 & \\
\hline & & & & \\
\hline
\end{tabular}

\section{SONUÇLAR ve TARTIŞMA}

Bu çalışmada, önemli jeodezik problemlerden bir tanesi olan koordinat dönüșüm problemi EKK ve TEKK yaklaşımları kullanılarak incelenmiştir. Bunun için problem, yapay olarak üretilmiş 2 boyutlu ağlarda test edilmiştir. 2 boyutlu ağa ait nokta koordinatları doğrultu-kenar ölçülerinden hesaplanmıștır. İkinci datuma ait koordinatlar, her bir durum için tablolarda verilen değerler kullanılarak orijinal dönüşüm parametrelerinden üretilmiştir. Koordinat dönüşümü jeodezik problemlere sıklıkla uygulanmasına rağmen, son yillarda yaygin olarak kullanılmaya başlayan TEKK yöntemine ait doğruluk analizleri henüz incelenmemiştir. Modele ait doğruluk analizlerinin tek bir üretilen ağdan yapılması yeterli olmayacağından dolayı, yöntemin performansını değerlendirmek için 10000 adet ağ oluşturulmuştur. Her durum için, kestirilen parametreler orijinal 
parametrelerle karşıllaştırılmış ve norm değerleri hesaplanmıştır. TEKK yaklaşımında, ölçüler ve katsayılar matrisi hatalı alınırsa, daha güvenilir sonuçlara ulaşılmaktadır. Ancak, EKK kestiriminde, sadece ölçü vektörünün hatalı olduğu kabul edilmektedir. $\mathrm{Bu}$ durum, katsay1lar matrisinin ve ölçü vektörünün hata içerdiği bu deneysel çalışmada da gözlemlenmiştir. Bu bağlamda, TEKK yaklaşımının, koordinat dönüşümü için parametre kestiriminde uygulanmasının gerekliliği ortaya çıkmaktadır.

\section{KAYNAKÇA}

Akyılmaz, O. (2007). Total Least Squares Solution of Coordinate Transformation. Survey Review, 39, 303, 68-80.

Akyılmaz, O., Acar, M., Ozludemir, M. T. (2007) "Koordinat Dönüşümünde En Küçük Kareler ve Toplam En Küçük Kareler Yöntemleri", HKM Jeodezi, Jeoinformasyon ve Arazi Yönetimi Dergisi, Vol. 2007/2, No. 97, 15-21

Felus, Y. (2004). Application of Total Least Squares for Spatial Point Process Analysis. Journal of Surveying Engineering, 103 (3), 126-133.

Golub, H.G., Van Loan, F.C. (1980). An Analysis of the Total Least Squares Problem. SIAM Journal of Numerical Analysis, 17 (6), 883893.

Guobin Chang, G., Lin, P., Bian, H., ve Gao, J. (2018). Simultaneous Helmert transformations among multiple frames considering all relevant measurements. Meas. Sci. Technol. 29, 035801 (10pp)

Hekimoglu, S., ve Erenoglu, R. C. (2013). A new GM-estimate with high breakdown point. Acta Geodaetica et Geophysica, 48(4), 419-437

Markovsky, I., ve Van Huffel, S. (2007). Overview of total least-squares methods. Signal Processing, 87, 2283-2302.

Neitzel, F. (2010). Generalisation of total least squares on example of unweighted and weighted similarity transformation. $J$ Geodesy, 35, 751-762.

Schaffrin, B., Lee, I., Felus, Y., ve Choi, Y. (2006). Total least squares (TLS) for geodetic straight-line and plane adjustment. Boll Geod Sci Aff., 65 (3), 141-168.

Schaffrin, B., ve Wieser, A. (2008). On weighted total least-squares adjustment for linear regression. J. Geodes., 82(7), 415-21.
Van Huffel, S., ve Vandewalle, J. (1991). The total least squares problem, computational aspects and analysis. Society for Industrial and Applied Mathematics, Philadelphia. 